\title{
Estimation and Fault Diagnosis of Lithium-Ion Batteries: A Fractional-Order System Approach
}

\author{
Shulan Kong ${ }^{D},{ }^{1}$ Mehrdad Saif, ${ }^{2}$ and Guozeng Cui ${ }^{3}$ \\ ${ }^{1}$ School of Mathematics Science, Qufu Normal University, Qufu 273165, China \\ ${ }^{2}$ Department of Electrical and Computer Engineering, University of Windsor, Windsor, ON N9B 3P4, Canada \\ ${ }^{3}$ School of Electronic and Information Engineering, Suzhou University of Science and Technology, Suzhou 215009, China
}

Correspondence should be addressed to Shulan Kong; shulank@163.com

Received 20 July 2018; Revised 1 October 2018; Accepted 10 October 2018; Published 24 October 2018

Guest Editor: Hiroaki Mukaidani

Copyright (C) 2018 Shulan Kong et al. This is an open access article distributed under the Creative Commons Attribution License, which permits unrestricted use, distribution, and reproduction in any medium, provided the original work is properly cited.

\begin{abstract}
This study investigates estimation and fault diagnosis of fractional-order Lithium-ion battery system. Two simple and common types of observers are designed to address the design of fault diagnosis and estimation for the fractional-order systems. Fractionalorder Luenberger observers are employed to generate residuals which are then used to investigate the feasibility of model based fault detection and isolation. Once a fault is detected and isolated, a fractional-order sliding mode observer is constructed to provide an estimate of the isolated fault. The paper presents some theoretical results for designing stable observers and fault estimators. In particular, the notion of stability in the sense of Mittag-Leffler is first introduced to discuss the state estimation error dynamics. Overall, the design of the Luenberger observer as well as the sliding mode observer can accomplish fault detection, fault isolation, and estimation. The effectiveness of the proposed strategy on a three-cell battery string system is demonstrated.
\end{abstract}

\section{Introduction}

Lithium-ion (Li-ion) batteries are an integral part of electrified vehicles and have found many other areas of applications in consumer products, space, marine, and other applications. They however remain a costly and safety critical subsystem in many areas that they are used in. As a result, reliability, safety, and efficient operation of Li-ion batteries in high power applications, such as in electrified vehicles, and challenging problems such as modeling, state estimation, monitoring, diagnostics, and pyrognostics capabilities are critical areas for research [1-8].

With the development of natural science and complex engineering applications, it has been found that many realworld physical systems show fractional-order dynamical behavior. And fractional calculus theories have been increasingly applied to many practical systems of a variety of scientific and engineering fields. The first application of the fractional calculus was accomplished by Abel in 1823 while investigating the solution of the famous problem of tautochrone [9]. For almost two hundred years, fractionalorder models have been used to describe biology systems [10] in bioengineering, financial markets [11-13] in economics, and diffusion wave [14-17] and lead-acid battery or Li-ion battery [18-21] in physical engineering. Due to inherent properties of fractional-order calculus, fractional-order differential systems sometimes have a more appropriate mathematical description than integer-order differential systems for managing lithium-ion batteries. In integer-order models of Li-ion batteries, constant phase elements are either approximated by ideal capacitors, or a number of resistor-capacitor networks, or relaxation times [21]. The resulting models are commonly not capable of predicting battery dynamics in both the time and frequency domains over the entire operating range. They can not capture the key and accuracy battery characteristics. To address the above problems, a fractionalorder model of replacing the ideal capacitor in the first-order resistor-capacitor networks model to a fractional element is explored for Li-ion cells. By using experimental data from time and frequency domains, Alavi et al. [22] found that this model can reproduce a Li-ion battery's behavior better than its integer counterpoint, thanks to an additional degree of freedom, namely, the fractionation order. A fractional-order state space model of lithium-ion battery was proposed and 
the experiment results showed that it has a better fitness than the classical equivalent circuit models based on the integer differential equations in [20]. In addition, a comprehensive review of fractional-order techniques for $\mathrm{Li}$-ion batteries was referred to [21]. Consequently, the fractional-order modeling methodology may not only improve prediction accuracy but also preserve some physical meanings underlying model parameters.

Catastrophic failures and/or explosion of Li-ion batteries in various applications ranging from cell phones to aircrafts and automobiles are all familiar new stories of the past few years. It is therefore imperative that, at the onset of failures, such events are detected and preventive measures to be taken [23-25]. Model based fault detection and isolation strategies could prove invaluable in this direction. For such techniques to be effective, availability of accurate representative model of the system is necessary. Accordingly, significant progress towards fault diagnosis and estimation of Li-ion batteries modeled as integer-order differential systems have recently been made in $[1-7,26-28]$ and the references therein. In particular a synthesized design of reduced-order Luenberger observers and Learning observers was presented for the purpose of simultaneous fault isolation and estimation of a three-cell battery string in [1]. As discussed in the previous paragraph, Li-ion battery systems do exhibit the fractional phenomena. The fractional characteristic could give a better description of the mathematical model of the battery system and can potentially lead to more effective fault detection and estimation strategies. Inspired by the abovementioned works and observation, a fractional-order system approach will be considered for estimation and fault diagnosis of fractional-order Li-ion battery system instead of the integerorder system [1].

Observer-based fault diagnosis of fractional-order systems remains problematic due to the lack of well-established and reliable techniques even though the design of observers for fractional-order systems has been reported [29-33]. However, little progress on fault detection and estimation for fractional-order models has been made besides [34, 35]. Reference [35] aims to provide sufficient conditions of the asymptotical convergence for the fractional-order state estimation errors and fault estimation errors via a frequencydistributed fractional integrator equivalent model [36] and employs an indirect Lyapunov method $[30,37,38]$. Recently observer-based tracking control of fractional-order systems is addressed by using Mittag-Leffler function based Lyapunov methods to prove the boundedness of state estimation error in [33]. As such, it is of practical and theoretical importance to focus studies on observer-based fault diagnosis of Li-ion batteries expressed by the fractional-order differential systems.

The main goal of this paper is to propose a strategy for fault detection, isolation, and estimation via fractional-order differential models. The goal is not only in detecting, and if possible isolating and estimating the fault in Li-ion battery system, but also in theoretical development for designing stable observers and estimating faults. Firstly, sources of faults and uncertainties are separated by reorganizing the original system. Towards this, the system is divided into two subsystems. This will allow us to give detectability conditions under which a stable fractional-order observer exists for the second subsystem. A fault-detection residual is then generated by constructing a fractional-order Luenberger observer. The residual is used to characterize the feasibility of fault detection and isolation. Once a fault occurs in the system, it is possible to detect and isolate it. After that, we attempt to further explore the isolated fault. In this step, a fractional-order sliding-mode observer is designed to provide an estimate of the isolated fault for the first subsystem containing the isolated fault as well as the systems uncertainties. MittagLeffler stability of fractional-order estimation error system is defined and the estimation error is proved to be MittagLeffler stable. As a result, the synthesized design of the fractional-order Luenberger observers and the fractionalorder sliding-mode observer can lead to simultaneous fault isolation and estimation.

The main contribution of the paper is twofold. On one hand, two simple and common types of fractional-order observer-based strategies are derived to satisfactorily accomplish the task of fault detection, isolation, and estimation for the fractional-order Li-ion battery system inspired by the ideas proposed in $[1,39,40]$. On the other hand, MittagLeffler stability of fractional-order estimation error system is defined and some other theoretical results are then presented. It is believed that the obtained results can perfectly embody accuracy and practicability properties of fractional calculus describing a real process in physical systems.

The rest of the paper is organized as follows. In Section 2, a fractional-order system based on a Li-ion battery model is formulated to achieve fault diagnosis and estimation and to answer the listed three questions. A strategy for fault detection and isolation is presented in Section 3 and fault estimation strategy is given in Section 4. In Section 5 the proposed strategy is applied to a three-cell battery string system to show the effectiveness of the proposed approach. Finally, some conclusions are drawn in Section 6.

\section{Problem Formulation}

Inspired by the idea of [20], we are ready to present a fractional-order model for a general system in [1]. Consider the following fractional-order pseudostate space description

$$
\begin{aligned}
& D^{\alpha} x(t)=A x(t)+B u(t)+F f(t)+G d(t), \\
& 0<\alpha<1 \text {, } \\
& y(t)=C x(t)
\end{aligned}
$$

where $D^{\alpha}$ denotes the Caputo fractional derivative of order $\alpha$ on $[0, t], \alpha$ is the fractional commensurate order, $x \in R^{n}$ is the pseudostate vector, $u \in R^{m}$ is the input vector, $y \in$ $R^{p}$ is the measurable output vector, $f(t)$ is a bounded $s-$ dimensional fault vector, $d(t)$ represents bounded uncertainties/disturbances with dimension of $q$, and $A, B, F, G$, and $C$ are $n \times n, n \times m, n \times s, n \times q$, and $p \times n$ constant matrices, respectively.

Define $F=\left[\begin{array}{llll}F_{1} & F_{2} & \cdots & F_{s}\end{array}\right]$ and $G=\left[\begin{array}{llll}G_{1} & G_{2} & \cdots & G_{q}\end{array}\right]$ for any $i \in\{1,2, \ldots, s\}$ and $j \in\{1,2, \ldots, q\}$, and $F_{i}$ and $G_{j}$ are the $i$-th and $j$-th column of $F$ and $G$. Denote also $G_{i}{ }^{\prime}$ as a matrix consisting of $F_{i}$ and all columns of $G$, that is, $G_{i}{ }^{\prime}=$ $\left[\begin{array}{lllll}F_{i} & G_{1} & G_{2} & \cdots & G_{q}\end{array}\right]$ for any $i \in\{1,2, \ldots, s\}$. 
For the above system, the following assumptions are made.

Assumption 1. At most a fault occurs at one time in system (1).

Assumption 2. Matrix $G$ is of full column $\operatorname{rank}, \operatorname{rank}(C)=p$, and $\operatorname{rank}\left(C G_{i}{ }^{\prime}\right)=\operatorname{rank}\left(G_{i}{ }^{\prime}\right)=q+1$ where $\operatorname{rank}(\cdot)$ denotes the rank of a matrix.

Assumption 3. For each $i=1,2, \ldots, s$ and for every complex number $\sigma$,

$$
\operatorname{rank}\left[\begin{array}{cc}
\sigma I_{n}-A & G_{i}^{\prime} \\
C & 0
\end{array}\right]=n+q+1
$$

with $|\arg (\sigma)| \leq \alpha \pi / 2, I_{n}$ is the $n \times n$ identity matrix, and $\arg (\sigma)$ stands for the argument of $\sigma$.

Remark 4. Assumption 2 implies that $G_{i}{ }^{\prime}$ is of full column rank and $q+1 \leq p$. Then $F$ has no zero columns.

The purpose of this paper is to study the fault diagnosis problem. The fault diagnosis problem addressed here is to detect, isolate, and estimate the fault. A systematic study is performed to answer the three following questions under Assumptions 1-3:

(1) How can a fault be detected or what are the possible conditions for detecting a fault when it occurs?

(2) How can the detected fault be isolated?

(3) How can the isolated fault be dealt with or estimated?

\section{Fault Detection and Isolation}

In the section, we will reorganize system (1) and develop an approach to fault diagnosis. To present the conditions for fault detection and isolation, a particular system structure is presented, which is the structure to be built on the following results.

Lemma 5. $\operatorname{Rank}\left(C G_{i}{ }^{\prime}\right)=q+1$ if and only if there exist nonsingular matrices $T_{i}$ and $S_{i}$ such that

$$
\begin{aligned}
T_{i}^{-1} G_{i}{ }^{\prime} & =\left[\begin{array}{c}
G_{i}^{1} \\
0
\end{array}\right], \\
S_{i}^{-1} C T_{i} & =\left[\begin{array}{cc}
C_{i}^{11} & 0 \\
0 & C_{i}^{22}
\end{array}\right],
\end{aligned}
$$

where $G_{i}^{1}$ and $C_{i}^{11}$ are invertible, $\operatorname{rank}\left(G_{i}^{1}\right)=\operatorname{rank}\left(C_{i}^{11}\right)=q+1$.

Proof. Based on Lemma 1 presented in [41], it is obtained that $\operatorname{rank}\left(C G_{i}{ }^{\prime}\right)=\operatorname{rank}\left(G_{i}{ }^{\prime}\right)$ if and only if there are nonsingular matrices $T_{i}$ and $S_{i}$ such that

$$
\begin{aligned}
T_{i}^{-1} G_{i}^{\prime} & =\left[\begin{array}{c}
G_{i}^{1} \\
0
\end{array}\right], \\
S_{i}^{-1} C T_{i} & =\left[\begin{array}{cc}
C_{i}^{11} & 0 \\
0 & C_{i}^{22}
\end{array}\right],
\end{aligned}
$$

where $G_{i}^{1}$ and $C_{i}^{11}$ have the same number of rows, $G_{i}^{1}$ is of full row rank, and $C_{i}^{11}$ is invertible.

Considering Assumption 2 and

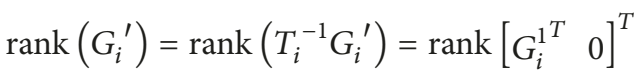

$$
\begin{aligned}
& =\operatorname{rank}\left(G_{i}^{1}\right) \text {, }
\end{aligned}
$$

where $T$ means the transpose of a matrix, we can obtain that $G_{i}^{1}$ is of full column $\operatorname{rank}$ and $\operatorname{rank}\left(G_{i}^{1}\right)=q+1$. It is worth noting that $G_{i}^{1}$ is also of full row rank, and then $G_{i}^{1}$ is invertible. Because $C_{i}^{11}$ is invertible, and $G_{i}^{1}$ and $C_{i}^{11}$ have the same number of rows, then $\operatorname{rank}\left(C_{i}^{11}\right)=q+1$.

Remark 6. Lemma 5 is equivalent to the existence of state and output transformations. In order to effectively isolate every fault, the original system (1) can be reorganized using Lemma 5.

3.1. Bank of Reformulated Systems. This subsection deals with transformation of the system into a proper form containing two subsystems. Define $f(t)=\left[\begin{array}{llll}f_{1}(t) & f_{2}(t) & \cdots & f_{s}(t)\end{array}\right]^{T}, \widetilde{F}_{i}$ is defined as the matrix after removing the column $F_{i}$ from $F$, and $\bar{f}_{i}(t)$ is the matrix after deleting the $f_{i}(t)$ from $f(t)$. Then, system (1) is reformulated as a bank of $s$ systems as follows:

$$
\begin{aligned}
D^{\alpha} x(t)= & A x(t)+B u(t)+\widetilde{F}_{i} \bar{f}_{i}(t)+F_{i} f_{i}(t) \\
& +G d(t), \\
y(t)= & C x(t), \quad i=1,2, \ldots, s .
\end{aligned}
$$

According to Lemma 5, for $i \in\{1,2, \ldots, s\}$, there exist nonsingular matrices $T_{i}$ and $S_{i}$ such that state and output transformations are described as

$$
\begin{aligned}
& x=T_{i}\left[\begin{array}{l}
\xi_{i}^{1} \\
\xi_{i}^{2}
\end{array}\right], \\
& y=S_{i}\left[\begin{array}{l}
\eta_{i}^{1} \\
\eta_{i}^{2}
\end{array}\right]
\end{aligned}
$$

and (6) can be correspondingly transformed as

$$
\begin{aligned}
D^{\alpha} \xi_{i}^{1}(t)= & A_{i}^{11} \xi_{i}^{1}(t)+A_{i}^{12} \xi_{i}^{2}(t)+B_{i}^{1} u(t)+\bar{F}_{i}^{1} \bar{f}_{i}(t) \\
& +G_{i}^{1}\left[\begin{array}{l}
f_{i}(t) \\
d(t)
\end{array}\right] \\
D^{\alpha} \xi_{i}^{2}(t)= & A_{i}^{21} \xi_{i}^{1}(t)+A_{i}^{22} \xi_{i}^{2}(t)+B_{i}^{2} u(t)+\bar{F}_{i}^{2} \bar{f}_{i}(t) \\
\eta_{i}^{1}(t)= & C_{i}^{11} \xi_{i}^{1}(t) \\
\eta_{i}^{2}(t)= & C_{i}^{22} \xi_{i}^{2}(t),
\end{aligned}
$$

where $G_{i}^{1}$ and $C_{i}^{11}$ are $(q+1) \times(q+1)$ matrices, $\xi_{i}^{1}(t)$ and $\xi_{i}^{2}(t)$ are $(q+1)$-dimensional and $(n-q-1)$-dimensional vectors, and 


$$
\begin{aligned}
T_{i}^{-1} A T_{i} & =\left[\begin{array}{cc}
A_{i}^{11} & A_{i}^{12} \\
A_{i}^{21} & A_{i}^{22}
\end{array}\right], \\
T_{i}^{-1} B & =\left[\begin{array}{c}
B_{i}^{1} \\
B_{i}^{2}
\end{array}\right], \\
T_{i}^{-1} \widetilde{F}_{i} & =\left[\begin{array}{c}
\bar{F}_{i}^{1} \\
\bar{F}_{i}^{2}
\end{array}\right], \\
T_{i}^{-1} G_{i}{ }^{\prime} & =\left[\begin{array}{c}
G_{i}^{1} \\
0
\end{array}\right], \\
S_{i}^{-1} C T_{i} & =\left[\begin{array}{cc}
C_{i}^{11} & 0 \\
0 & C_{i}^{22}
\end{array}\right],
\end{aligned}
$$

where the dimensions of matrices are defined as follows: $A_{i}^{11}$ is $(q+1) \times(q+1), A_{i}^{12}$ is $(q+1) \times(n-q-1), A_{i}^{21}$ is $(n-q-1) \times(q+1)$, $A_{i}^{22}$ is $(n-q-1) \times(n-q-1), \bar{F}_{i}^{1}$ is $(q+1) \times(s-1), \bar{F}_{i}^{2}$ is $(n-q-1) \times(s-1)$, and $C_{i}^{22}$ is $(p-q-1) \times(n-q-1)$.

System (8) can be rewritten as two subsystems

$$
\begin{aligned}
D^{\alpha} \xi_{i}^{1}(t)= & A_{i}^{11} \xi_{i}^{1}(t)+A_{i}^{12} \xi_{i}^{2}(t)+B_{i}^{1} u(t)+\bar{F}_{i}^{1} \bar{f}_{i}(t) \\
& +G_{i}^{1}\left[\begin{array}{c}
f_{i}(t) \\
d(t)
\end{array}\right] \\
\eta_{i}^{1}(t)= & C_{i}^{11} \xi_{i}^{1}(t), \quad i=1,2, \ldots, s
\end{aligned}
$$

and

$$
\begin{aligned}
D^{\alpha} \xi_{i}^{2}(t) & =A_{i}^{21} \xi_{i}^{1}(t)+A_{i}^{22} \xi_{i}^{2}(t)+B_{i}^{2} u(t)+\bar{F}_{i}^{2} \bar{f}_{i}(t) \\
\eta_{i}^{2}(t) & =C_{i}^{22} \xi_{i}^{2}(t), \quad i=1,2, \ldots, s,
\end{aligned}
$$

where all variables and matrices here are the same as before.

Remark 7. It is important to separate a single fault source and disturbances simultaneously by introducing state and output transformations. As well known, the key of the fault isolation is to isolate the single fault source.

3.2. Fault Detection Based on a Luenberger Observer. The observer technique is used to detect and isolate faults in this subsection. We propose to construct an observer for the second subsystem (11) for each $i$.

Lemma 8. Consider (11) satisfying Assumptions 2 and 3; then

$$
\operatorname{rank}\left(\left[\begin{array}{c}
\sigma I_{n-q-1}-A_{i}^{22} \\
C_{i}^{22}
\end{array}\right]\right)=n-q-1 .
$$

Proof. By performing elementary column and row operations on matrix and using Lemma 5 , the following equivalence is shown:

$$
\left[\begin{array}{cc}
\sigma I_{n}-A & G_{i}^{\prime} \\
C & 0
\end{array}\right] \sim\left[\begin{array}{ccc}
G_{i}^{1} & 0 & 0 \\
0 & C_{i}^{11} & 0 \\
0 & 0 & \sigma I_{n-q_{-1}}-A_{i}^{22} \\
0 & 0 & C_{i}^{22}
\end{array}\right]
$$

where " $\sim$ " means equivalence. It is worth noting that

$$
\begin{aligned}
& \operatorname{rank}\left(\left[\begin{array}{cc}
\sigma I_{n}-A & G_{i}^{\prime} \\
C & 0
\end{array}\right]\right) \\
& \quad=\operatorname{rank}\left(\left[\begin{array}{c}
\sigma I_{n-q-1}-A_{i}^{22} \\
C_{i}^{22}
\end{array}\right]\right)+2(q+1),
\end{aligned}
$$

and

$$
\operatorname{rank}\left(\left[\begin{array}{cc}
\sigma I_{n}-A & G_{i}^{\prime} \\
C & 0
\end{array}\right]\right)=n+(q+1),
$$

And then (12) is obtained.

Meanwhile, we can obtain a conclusion in Theorem 9 similar to Lemma 1 presented in [42].

Theorem 9. With Assumptions 2 and 3, the unobservable polynomial of the pair $\left(A_{i}^{22}, C_{i}^{22}\right)$ is equal to the invariant zero polynomial of $\left(C, A, G_{i}^{\prime}\right)$.

Following Lemmas 2 and 3 in [31], Theorem 10 can be stated.

Theorem 10. System (11) satisfying Assumptions 2 and 3 is detectable or the pair $\left(A_{i}^{22}, C_{i}^{22}\right)$ is detectable.

Considering system (11), $\xi_{i}^{1}$ and $\eta_{i}^{2}$ can be obtained from the measured output $y$. In fact, let

$$
S_{i}^{-1}=\left[\begin{array}{c}
S_{i}^{1} \\
S_{i}^{2}
\end{array}\right]
$$

And then they are computed by

$$
\begin{aligned}
\xi_{i}^{1} & =\left(C_{i}^{11}\right)^{-1} S_{i}^{1} y, \\
\eta_{i}^{2} & =S_{i}^{2} y .
\end{aligned}
$$

Moreover, based on Theorem 2 presented in [43] and Theorem 10 in this work, we are ready to design a Luenberger observer for system (11) and select $L_{i}^{2}$ such that $\left(A_{i}^{22}-L_{i}^{2} C_{i}^{22}\right)$ is a stable matrix; that is, the spectrum of $\left(A_{i}^{22}-L_{i}^{2} C_{i}^{22}\right)$ can be assigned anywhere in the complex of region of asymptotic stability $|\arg (\sigma)|>\alpha \pi / 2$. A Luenberger observer is designed as follows:

$$
\begin{aligned}
D^{\alpha} \widehat{\xi}_{i}^{2}(t)= & A_{i}^{21} \xi_{i}^{1}(t)+A_{i}^{22} \widehat{\xi}_{i}^{2}(t)+B_{i}^{2} u(t) \\
& +L_{i}^{2}\left(\eta_{i}^{2}(t)-\widehat{\eta}_{i}^{2}(t)\right), \\
\widehat{\eta}_{i}^{2}(t)= & C_{i}^{22} \widehat{\xi}_{i}^{2}(t), \quad i=1,2, \ldots, s,
\end{aligned}
$$

where ${ }^{\wedge}$ indicates estimate. 
For each $i=1,2, \ldots, s$, the state estimation error dynamics of the second subsystem (11) can be obtained by subtracting (18) from (11)

$$
D_{t}^{\alpha} e_{i}^{2}(t)=\left(A_{i}^{22}-L_{i}^{2} C_{i}^{22}\right) e_{i}^{2}(t)+\bar{F}_{i}^{2} \bar{f}_{i}(t),
$$

where $e_{i}^{2}(t)=\xi_{i}^{2}(t)-\widehat{\xi}_{i}^{2}(t)$.

We now define a fault-detection residual as

$$
r_{i}(t)=\left\|\eta_{i}^{2}(t)-\hat{\eta}_{i}^{2}(t)\right\|=\left\|C_{i}^{22} e_{i}^{2}(t)\right\|,
$$

and then we have Theorem 11.

Theorem 11. For system (1) satisfying Assumptions 1-3 with a bank of Luenberger observers in the form of (18), we consider only one single fault scenario. If there exist $i, j$ and $i \neq j$ such that $\lim _{t \rightarrow \infty} r_{i}(t)=0$ and $\lim _{t \rightarrow \infty} r_{j}(t)=0, i, j \in$ $\{1,2, \ldots, s\}$, then the system is healthy. If there are $i, j$ such that $\lim _{t \rightarrow \infty} r_{i}(t)=0$ and $\lim _{t \rightarrow \infty} r_{j}(t) \neq 0$ for $j \neq i$, then $f_{i}(t) \neq 0$; that is, $f_{i}(t)$ is the only one fault.

Proof. If $\lim _{t \rightarrow \infty} r_{i}(t)=0$ and $\lim _{t \rightarrow \infty} r_{j}(t)=0$ when $i \neq j$, it follows that $\bar{f}_{i}(t) \equiv 0$ and $\bar{f}_{j}(t) \equiv 0$ from (20) and the stability of $\left(A_{i}^{22}-L_{i}^{2} C_{i}^{22}\right)$ in (19), it implies that the system is free from faults, and thus system (1) is healthy.

If $\lim _{t \rightarrow \infty} r_{i}(t)=0$, it holds that $\bar{f}_{i}(t) \equiv 0$ from (20) and the stability of $\left(A_{i}^{22}-L_{i}^{2} C_{i}^{22}\right)$ of (19). If $\lim _{t \rightarrow \infty} r_{j}(t) \neq 0$ when $j \neq i$, it follows that $\bar{F}_{j}^{2} \bar{f}_{j}(t) \neq 0$ from (19) and (20). Moreover, $\bar{f}_{j}(t)$ has one and only one nonzero element, which is $f_{i}(t)$. Thus, $f_{i}(t) \neq 0$ and $f_{j}(t)=0$ for all $j, j \neq i$.

Remark 12. Theorem 11 implies that the residual $r_{i}(t)$ is not sensitive to the $i$ th fault $f_{i}(t)$ but is sensitive to all other faults.

3.3. Fault Isolation Based on Fault-Detection Residuals. Based on the analysis presented in the above subsections, the following fault-detection and isolation algorithm was suggested.

Step 1. Design a bank of Luenberger observers of the form (18) for $i=1,2, \ldots, s$.

Step 2. Compute the $s$ fault-detection residuals $r_{i}(t), i=$ $1,2, \ldots, s$.

Step 3. Choose a threshold $r_{0}$ (which can be chosen as small as possible theoretically because $\lim _{t \rightarrow \infty} r_{i}(t)=0$ when $\left.f_{i}(t) \neq 0\right)$.

Step 4. If all residuals $r_{i}(t), i=1,2, \ldots, s$ are below the threshold, then, the system is healthy. If there is only one residual, say $r_{i_{0}}(t)$ with $i_{0} \in\{1,2, \ldots, s\}$, which is below the threshold, then it is claimed that a fault has been detected and the fault is isolated as $f_{i_{0}}(t)$.

Remark 13. Based on Theorem 11, a fault can be detected when system (1) has only one fault by computing at most $s$ fault-detection residuals $r_{i}(t)$ for the bank of Luenberger observers (18). Performing the above algorithm, the first two questions listed in Section 2 are addressed.

\section{Fault Estimation Based on Sliding Mode Observers}

In this section, fault estimation based on sliding mode observers is addressed and will address the third question posed above. It is assumed that a fault has been detected and isolated as $f_{i_{0}}(t)$.

4.1. Design of a Sliding Mode Observer for Fault Estimation. When the fault $f_{i_{0}}(t)$ occurs, $\lim _{t \rightarrow \infty} r_{i_{0}}(t)=0$ and $\bar{f}_{i_{0}}(t)=$ 0 . For convenience, the isolated fault $f_{i_{0}}(t)$ is still denoted as $f_{i}(t)$. The $i_{0}-t h$ is denoted as $i-t h$. In fact, the first subsystem (10) and the state estimation error dynamics (19) with fault $f_{i_{0}}(t)$ can be described as follows:

$$
\begin{aligned}
D^{\alpha} \xi_{i}^{1}(t)= & A_{i}^{11} \xi_{i}^{1}(t)+A_{i}^{12} \xi_{i}^{2}(t)+B_{i}^{1} u(t) \\
& +G_{i}^{1}\left[\begin{array}{l}
f_{i}(t) \\
d(t)
\end{array}\right] \\
\eta_{i}^{1}(t)= & C_{i}^{11} \xi_{i}^{1}(t), \quad i=1,2, \ldots, s
\end{aligned}
$$

and

$$
D_{t}^{\alpha} e_{i}^{2}(t)=\left(A_{i}^{22}-L_{i}^{2} C_{i}^{22}\right) e_{i}^{2}(t) .
$$

For system (21), the following equations are established:

$$
\begin{array}{r}
\operatorname{rank}\left(C_{i}^{11} G_{i}^{1}\right)=\operatorname{rank}\left(G_{i}^{1}\right)=q+1, \\
\operatorname{rank}\left(\left[\begin{array}{cc}
\sigma I_{q+1}-A_{i}^{11} & G_{i}^{1} \\
C_{i}^{11} & 0
\end{array}\right]\right)=q+1+\operatorname{rank}\left(G_{i}^{1}\right)
\end{array}
$$

based on Lemma 5 and the equivalence of the following two matrices:

$$
\left[\begin{array}{cc}
\sigma I_{q+1}-A_{i}^{11} & G_{i}^{1} \\
C_{i}^{11} & 0
\end{array}\right] \sim\left[\begin{array}{cc}
0 & G_{i}^{1} \\
C_{i}^{11} & 0
\end{array}\right]
$$

The stability of the fractional-order observer for subsystem (21) can be formulated by the two conditions (23) and (24) according to [35]. Parallel to the $i$ th Luenberger observer of the form (18), a sliding mode observer can be designed to estimate the fault for (21) as follows:

$$
\begin{aligned}
D^{\alpha} \widehat{\xi}_{i}^{1}(t)= & A_{i}^{11} \widehat{\xi}_{i}^{1}(t)+A_{i}^{12} \widehat{\xi}_{i}^{2}(t)+B_{i}^{1} u(t) \\
& +L_{i}^{1}\left(\eta_{i}^{1}(t)-\widehat{\eta}_{i}^{1}(t)\right)+G_{i}^{1} \widehat{v}_{i}(t) \\
\widehat{\eta}_{i}^{1}(t)= & C_{i}^{11} \widehat{\xi}_{i}^{1}(t), \quad i=1,2, \ldots, s,
\end{aligned}
$$

where $\widehat{v}_{i}(t)$ is the estimate of $v_{i}(t)=\left[f_{i}(t) d(t)\right]^{T}$. It is defined as

$$
\widehat{v}= \begin{cases}\rho_{i} \frac{K_{i}^{1} e_{\eta_{i}}}{\left\|K_{i}^{1} e_{\eta_{i}}\right\|}, & \left\|K_{i}^{1} e_{\eta_{i}}\right\| \neq 0 ; \\ 0, & \left\|K_{i}^{1} e_{\eta_{i}}\right\|=0\end{cases}
$$

with $e_{\eta_{i}}=\eta_{i}^{1}-\widehat{\eta}_{i}^{1}$ and constant matrix $K_{i}^{1} \in R^{(q+1) \times(q+1)}, \rho_{i}$ should be chosen to be a large enough constant, and $L_{i}^{1}$ can 
be selected such that the spectrum of $\left(A_{i}^{11}-L_{i}^{1} C_{i}^{11}\right)$ is a stable matrix.

Let $e_{i}^{1}(t)=\xi_{i}^{1}(t)-\hat{\xi}_{i}^{1}(t)$ be the estimation error of state $\xi_{i}^{1}(t)$, then the fractional-order state estimation error can be described as follows:

$$
D^{\alpha} e_{i}^{1}=\left(A_{i}^{11}-L_{i}^{1} C_{i}^{11}\right) e_{i}^{1}+A_{i}^{12} e_{i}^{2}+G_{i}^{1}(v-\widehat{v}),
$$

and $e_{\eta_{i}}=\eta_{i}^{1}-\widehat{\eta}_{i}^{1}=C_{i}^{11} e_{i}^{1}$.

4.2. Stability of the Designed Sliding Mode Observer. Before embarking on the fundamental theorem, we first give some preliminary results on Caputo fractional derivatives.

Definition 14 (see [9]). The two-parameter Mittag-Leffler function is defined by

$$
E_{\alpha, \beta}(z)=\sum_{k=0}^{\infty} \frac{z^{k}}{\Gamma(\alpha k+\beta)}, \quad \alpha>0, \beta>0, z \in C,
$$

where $\Gamma(\cdot)$ is the Gamma function with

$$
\Gamma(x)=\int_{0}^{\infty} t^{x-1} e^{-t} d t .
$$

When $\beta=1$, one has $E_{\alpha}(z)=E_{\alpha, 1}(z)$.

Let $e_{i}(t)=\left[\begin{array}{ll}e_{i}^{1}(t) & e_{i}^{2}(t)\end{array}\right]^{T}, e_{i}(0)=\left[\begin{array}{ll}0 & 0\end{array}\right]^{T}$. According to the definition in [44], $[0,0]^{T}$ is the equilibrium point of the following dynamic system:

$$
\begin{aligned}
& D^{\alpha} e_{i}^{1}(t)=\left(A_{i}^{11}-L_{i}^{1} C_{i}^{11}\right) e_{i}^{1}(t)+A_{i}^{12} e_{i}^{2}(t) \\
& D_{t}^{\alpha} e_{i}^{2}(t)=\left(A_{i}^{22}-L_{i}^{2} C_{i}^{22}\right) e_{i}^{2}(t) .
\end{aligned}
$$

Then we present the definition of stability for the system formed by (28) and (22) in the sense of Mittag-Leffler similar to those in $[44,45]$ as follows.

Definition 15. The solution $e_{i}(t)$ of the system formed by (28) and (22) is said to be Mittag-Leffler stable with respect to the equilibrium point $e_{i}(0)$ of system (31) if

$$
\begin{aligned}
& \left\|e_{i}(t)\right\| \leq\left\{m\left[e_{i}\left(t_{0}\right)\right] E_{\alpha}\left(-\lambda\left(t-t_{0}\right)^{\alpha}\right)\right\}^{b}, \\
& \alpha \in(0,1) \text {, }
\end{aligned}
$$

where $t_{0}=0$ is the initial time, $\lambda>0, b>0, m(0)=$ $0, m\left(e_{i}\right) \geq 0$, and $m\left(e_{i}\right)$ is locally Lipschitz with Lipschitz constant.

Lemma 16 (see [46]). Let $x(t) \in R^{n}$ be a differentiable vector; then, for any time instant,

$$
\frac{1}{2} D^{\alpha}\left(x^{T}(t) P x(t)\right) \leq x^{T}(t) P D^{\alpha} x(t),
$$

$$
\forall \alpha \in(0,1], t \geq 0,
$$

where $P \in R^{n \times n}$ is a positive definite constant matrix.
The following theorem is to present the stability of the designed observers in the sense of Mittag-Leffler.

Theorem 17. Consider (1) satisfying Assumptions 1-3, $f(t)$ and $d(t)$ are bounded, and the Luenberger observers and the sliding mode observer are designed in (18), (26), and (27). For $i=1,2, \ldots, s$, if there exist matrices $L_{i}^{1}, L_{i}^{2}, K_{i}^{1}$ and positive definite matrices $P_{1}, P_{2}, Q_{i}^{1}, Q_{i}^{2}$ such that

$$
\begin{aligned}
-Q_{i}^{1}= & \left(A_{i}^{11}-L_{i}^{1} C_{i}^{11}\right)^{T} P_{1}+P_{1}\left(A_{i}^{11}-L_{i}^{1} C_{i}^{11}\right)+P_{1}^{2} \\
-Q_{i}^{2}= & \left(A_{i}^{22}-L_{i}^{2} C_{i}^{22}\right)^{T} P_{2}+P_{2}\left(A_{i}^{22}-L_{i}^{2} C_{i}^{22}\right) \\
& +A_{i}^{12^{T}} A_{i}^{12} \\
P_{1} G_{i}^{1}= & C_{i}^{11^{T}} K_{i}^{1^{T}}
\end{aligned}
$$

then the observers given by (18), (26), and (27) can ensure that the state estimation error $e_{i}(t)$ is Mittag-Leffler stable.

Proof. Consider a Lyapunov function candidate as follows:

$$
V_{i}\left(t, e_{i}(t)\right)=e_{i}^{1^{T}}(t) P_{1} e_{i}^{1}(t)+e_{i}^{2^{T}}(t) P_{2} e_{i}^{2}(t),
$$

where $P_{1}$ and $P_{2}$ are positive definite matrices.

Applying Caputo fractional-order operation $D^{\alpha}$ to $V_{i}$ with respect to $t$ along (22) and (28), by Lemma 16 , it is obtained that

$$
\begin{aligned}
& D^{\alpha} V_{i}=D^{\alpha}\left(e_{i}^{1^{T}} P_{1} e_{i}^{1}\right)+D^{\alpha}\left(e_{i}^{2^{T}} P_{2} e_{i}^{2}\right) \\
& \quad \leq 2 e_{i}^{1^{T}} P_{1}\left(D^{\alpha} e_{i}^{1}\right)+2 e_{i}^{2^{T}} P_{2}\left(D^{\alpha} e_{i}^{1}\right) \\
& \quad=\left[\left(A_{i}^{11}-L_{i}^{1} C_{i}^{11}\right) e_{i}^{1}+A_{i}^{12} e_{i}^{2}+G_{i}^{1} e_{v}\right]^{T} P_{1} e_{i}^{1} \\
& \quad+e_{i}^{1^{T}} P_{1}\left[\left(A_{i}^{11}-L_{i}^{1} C_{i}^{11}\right) e_{i}^{1}+A_{i}^{12} e_{i}^{2}+G_{i}^{1} e_{v}\right] \\
& \quad+e_{i}^{2^{T}}\left[\left(A_{i}^{22}-L_{i}^{2} C_{i}^{22}\right)^{T} P_{2}+P_{2}\left(A_{i}^{22}-L_{i}^{2} C_{i}^{22}\right)\right] e_{i}^{2} \\
& \quad \leq e_{i}^{1^{T}}\left[\left(A_{i}^{11}-L_{i}^{1} C_{i}^{11}\right)^{T} P_{1}+P_{1}\left(A_{i}^{11}-L_{i}^{1} C_{i}^{11}\right)\right. \\
& \left.\quad+P_{1}^{2}\right] e_{i}^{1}+e_{i}^{2^{T}}\left[\left(A_{i}^{22}-L_{i}^{2} C_{i}^{22}\right)^{T} P_{2}\right. \\
& \left.\quad+P_{2}\left(A_{i}^{22}-L_{i}^{2} C_{i}^{22}\right)+A_{i}^{12^{T}} A_{i}^{12}\right] e_{i}^{2}+2 e_{i}^{1 T} P_{i}^{1} G_{i}^{1} e_{v},
\end{aligned}
$$

where $e_{v}=v-\widehat{v}$.

If $K_{i}^{1}$ can be chosen such that $P_{1} G_{i}^{1}=C_{i}^{11^{T}} K_{i}^{1^{T}}$, then

$$
\begin{aligned}
& e_{i}^{1^{T}} P_{1} G_{i}^{1} \widehat{v}=\left\|K_{i}^{1} C_{i}^{11} e_{i}^{1}\right\| \rho_{i}=\left\|K_{i}^{1} e_{\eta_{1}}\right\| \rho_{i}, \\
& e_{i}^{1^{T}} P_{1} G_{i}^{1} v=\left(K_{i}^{1} C_{i}^{11} e_{i}^{1}\right)^{T} v=\left(K_{i}^{1} e_{\eta_{1}}\right)^{T} v .
\end{aligned}
$$

Considering the following inequality:

$$
e_{i}^{1^{T}} P_{i}^{1} G_{i}^{1} v \leq\left\|K_{i}^{1} e_{\eta_{1}}\right\|\|v\|,
$$


and applying (35) and (36), (39) can be further manipulated as follows:

$$
\begin{gathered}
D^{\alpha} V_{i} \leq e_{i}^{1^{T}}\left(-Q_{i}^{1}\right) e_{i}^{1}+e_{i}^{2^{T}}\left(-Q_{i}^{2}\right) e_{i}^{2} \\
-2\left\|K_{i}^{1} e_{\eta_{1}}\right\|\left(\rho_{i}-\|v\|\right) .
\end{gathered}
$$

Let $Q_{i}=\left[\begin{array}{cc}Q_{i}^{1} & 0 \\ 0 & Q_{i}^{2}\end{array}\right]$ and $P=\left[\begin{array}{cc}P_{1} & 0 \\ 0 & P_{2}\end{array}\right], \lambda_{\min }\left(Q_{i}\right)$ denoted the minimum eigenvalue of $Q_{i}$, and $\lambda_{\text {min }}(P)$ and $\lambda_{\max }(P)$ denoted the minimum and maximum eigenvalues of $P$. Then $\lambda_{\text {min }}\left(Q_{i}\right)>0, \lambda_{\text {min }}(P)>0$, and $\lambda_{\text {max }}(P)>0$.

Choosing $\rho_{i}$ large enough (this can be performed because $v$ is bounded), one obtains

$$
D^{\alpha} V_{i} \leq-\lambda_{\min }\left(Q_{i}\right)\left\|e_{i}\right\|^{2} .
$$

And the Lyapunov function candidate is satisfied by

$$
\lambda_{\min }(P)\left\|e_{i}\right\|^{2} \leq V_{i} \leq \lambda_{\max }(P)\left\|e_{i}\right\|^{2} .
$$

It follows from (43) and (44) that

$$
D^{\alpha} V_{i} \leq-\frac{\lambda_{\min }\left(Q_{i}\right)}{\lambda_{\max }(P)} V_{i} .
$$

There exists a nonnegative function $M(t)$ such that

$$
D^{\alpha} V_{i}+M(t)=-\frac{\lambda_{\min }\left(Q_{i}\right)}{\lambda_{\max }(P)} V_{i} .
$$

Taking Laplace transform on both sides of (46) leads to

$$
s^{\alpha} V_{i}(s)-s^{\alpha-1} V_{i}(0)+M(s)=-\frac{\lambda_{\min }\left(Q_{i}\right)}{\lambda_{\max }(P)} V_{i}(s),
$$

where $V_{i}(s)=\mathfrak{f}\left\{V_{i}\left(t, e_{i}(t)\right)\right\}$ and $£$ is the operator for Laplace transform. Based on (47), $V_{i}$ can be expressed as follows:

$$
V_{i}(s)=\frac{s^{\alpha-1} V_{i}(0)-M(s)}{s^{\alpha}+\lambda_{\min }\left(Q_{i}\right) / \lambda_{\max }(P)} .
$$

From the existence and uniqueness theorem [9] for fractional-order differential equations and the inverse Laplace transform, the unique solution of (46) can be obtained as follows:

$$
\begin{aligned}
V_{i}(t)= & V_{i}(0) E_{\alpha}\left(-\frac{\lambda_{\text {min }}\left(Q_{i}\right)}{\lambda_{\max }(P)} t^{\alpha}\right)-M(t) \\
& *\left[t^{\alpha-1} E_{\alpha, \alpha}\left(-\frac{\lambda_{\min }\left(Q_{i}\right)}{\lambda_{\max }(P)} t^{\alpha}\right)\right],
\end{aligned}
$$

where $*$ denotes the convolution operator.

Therefore, $V_{i}(t) \leq V_{i}(0) E_{\alpha}\left(-\left(\lambda_{\text {min }}\left(Q_{i}\right) / \lambda_{\text {max }}(P)\right) t^{\alpha}\right)$ from the facts that both $t^{\alpha-1}$ and $E_{\alpha, \alpha}\left(-\left(\lambda_{\min }\left(Q_{i}\right) / \lambda_{\max }(P)\right) t^{\alpha}\right)$ are nonnegative. With the aid of $(44)$, the estimation error $e_{i}(t)$ satisfies the following inequality:

$$
\left\|e_{i}(t)\right\| \leq\left[\frac{V_{i}(0)}{\lambda_{\min }(P)} E_{\alpha}\left(-\frac{\lambda_{\min }\left(Q_{i}\right)}{\lambda_{\max }(P)} t^{\alpha}\right)\right]^{1 / 2},
$$

where $V_{i}(0) / \lambda_{\min }(P) \geq 0$.
Let $m=V_{i}(0) / \lambda_{\min }(P)=V_{i}\left(0, e_{i}(0)\right) / \lambda_{\min }(P) \geq 0$, and then we have

$$
\left\|e_{i}(t)\right\| \leq\left[m E_{\alpha}\left(-\frac{\lambda_{\min }\left(Q_{i}\right)}{\lambda_{\max }(P)} t^{\alpha}\right)\right]^{1 / 2},
$$

where $m=0$ holds if and only if $e_{i}(0)=0$. Because $V\left(t, e_{i}\right)$ is locally Lipschitz with respect to $e_{i}$ and $V\left(0, e_{i}(0)\right)=0$ if and only if $e_{i}(0)=0$, and it follows that $m=V_{i}\left(0, e_{i}(0)\right) / \lambda_{\min }(P)$ is also Lipschitz with respect to $e_{i}(0)$ and $m(0)=0$. Therefore, the solution $e_{i}(t)$ of the system formed by (28) and (22) is Mittag-Leffler stable.

Remark 18. Theorem 17 implies that the state estimation errors $e_{i}^{1}$ and $e_{i}^{2}$ are bounded when $E_{\alpha}\left(-\left(\lambda_{\min }\left(Q_{i}\right) /\right.\right.$ $\left.\lambda_{\text {max }}(P)\right) t^{\alpha}$ ) is bounded. The conclusion is the same as that for integer-order systems [1].

Remark 19. The third question in Section 2 has been addressed. To our knowledge the stability in the sense of Mittag- Leffler is first introduced here to study the state estimation error dynamics.

\section{Fault Detection and Estimation of the Three-Cell Battery String}

In this section, we apply the proposed method for fault detection and estimation of a three-cell battery string model reported in [1] and demonstrate its effectiveness in application. Then system (1) is now parameterized as follows:

$$
\begin{aligned}
A & =\left[\begin{array}{ccc}
-1.6026 & 0 & 0 \\
0 & -1.6026 & 0 \\
0 & 0 & -1.6026
\end{array}\right], \\
B & =\left[\begin{array}{lll}
0.1795 & 5.2484 & 0.0225 \\
0.1795 & 5.2484 & 0.0225 \\
0.1795 & 5.2484 & 0.0225
\end{array}\right], \\
C & =\left[\begin{array}{lll}
1 & 0 & 0 \\
0 & 1 & 0 \\
0 & 0 & 1
\end{array}\right], \\
G & =\left[\begin{array}{ll}
0.1 \\
1 \\
0.05
\end{array}\right], \\
F & =\left[\begin{array}{lll}
3 & 0 & 0 \\
0 & 3 & 0 \\
0 & 0 & 3
\end{array}\right] .
\end{aligned}
$$

A disturbance is selected as $d(t)=0.0151 \sin (5 t)$. It is assumed that $f_{1}=f_{3}=0$ for all the time. When $t \in[0, T)$, $f_{2}=0$ and when $t \geq T, f_{2}=0.2$; that is, fault $f_{2}$ occurs at time $T$. Furthermore, a threshold is set as $r_{0}=0.015$ and take $T=25 s$ where $s$ stands for "second." 
Because there are three fault sources, system (1) has three formulations with each one corresponding to a particular fault. According to Lemma 5, nonsingular transform matrices are selected as

$$
\begin{aligned}
& T_{1}=\left[\begin{array}{ccc}
1 & 0 & 0 \\
0 & 1 & 0 \\
0 & 0.05 & 1
\end{array}\right], \\
& T_{2}=\left[\begin{array}{lll}
1 & 0 & 0 \\
0 & 1 & 0 \\
0.5 & 0 & 1
\end{array}\right], \\
& T_{3}=\left[\begin{array}{lll}
0.1 & 0 & 1 \\
1 & 0 & 0 \\
0 & 1 & 0
\end{array}\right]
\end{aligned}
$$

and $S_{i}=T_{i}, \quad i=1,2,3$.

Obviously, for each $i$-th system, the second subsystem does not include $i$-th fault source $f_{i}$ and their observers are designed as

$$
\begin{aligned}
D^{\alpha} \xi_{1}^{2}(t)= & -1.6026 \xi_{1}^{2}(t)+0.1705 Z+4.9860 \\
& +0.0214 I-0.15 f_{2}+3 f_{3}, \\
\eta_{1}^{2}(t)= & \xi_{1}^{2}(t) ; \\
D^{\alpha} \widehat{\xi}_{1}^{2}(t)= & -1.6026 \hat{\xi}_{1}^{2}(t)+0.1705 Z+4.9860 \\
& +0.0214 I+0.6\left(\eta_{i}^{2}(t)-\widehat{\eta}_{i}^{2}(t)\right), \\
\hat{\eta}_{1}^{2}(t)= & \widehat{\xi}_{1}^{2}(t), \\
D^{\alpha} \xi_{2}^{2}(t)= & -1.6026 \xi_{2}^{2}(t)+0.0897 Z+2.6242 \\
& +0.0113 I-1.5 f_{1}+3 f_{3}, \\
\eta_{2}^{2}(t)= & \xi_{2}^{2}(t) ; \\
D^{\alpha} \hat{\xi}_{2}^{2}(t)= & -1.6026 \hat{\xi}_{2}^{2}(t)+0.0897 Z+2.6242 \\
& +0.0113 I+0.3\left(\eta_{2}^{2}(t)-\widehat{\eta}_{2}^{2}(t)\right), \\
\widehat{\eta}_{2}^{2}(t)= & \widehat{\xi}_{2}^{2}(t), \\
D^{\alpha} \xi_{3}^{2}(t)= & -1.6026 \xi_{3}^{2}(t)+0.1616 Z+4.7236 \\
& +0.0203 I+3 f_{1}-0.3 f_{2}, \\
& +0.0203 I+0.6\left(\eta_{3}^{2}(t)-\widehat{\eta}_{3}^{2}(t)\right), \\
\eta_{3}^{2}(t)= & \xi_{3}^{2}(t) ; \\
D^{\alpha} \widehat{\xi}_{3}^{2}(t)= & -1.6026 \hat{\xi}_{3}^{2}(t)+0.1616 Z+4.7236 \\
\hat{\eta}_{3}^{2}(t)= & \hat{\xi}_{3}^{2}(t),
\end{aligned}
$$

where $L_{1}^{2}=L_{3}^{2}=0.6, L_{2}^{2}=0.3$.
Then, the $i$-th fault-detection residual is designated as

$$
r_{i}(t)=\left\|\eta_{i}^{2}(t)-\widehat{\eta}_{i}^{2}(t)\right\|=\left\|e_{i}^{2}(t)\right\|, \quad i=1,2,3 .
$$

In what follows, we only show the first subsystem and its observer for $i=2$. Let

$$
\begin{aligned}
& \xi_{2}^{1}(t)=\left[\begin{array}{l}
\xi_{21}^{1}(t) \\
\xi_{22}^{1}(t)
\end{array}\right], \\
& \eta_{2}^{1}(t)=\left[\begin{array}{l}
\eta_{21}^{1}(t) \\
\eta_{22}^{1}(t)
\end{array}\right],
\end{aligned}
$$

and then the subsystem and its sliding mode observer are as follows:

$$
\begin{aligned}
D^{\alpha} \xi_{21}^{1}(t)= & -1.6026 \xi_{21}^{1}(t)+0.1795 Z+5.2484 \\
& +0.0225 I+0.1 d(t) \\
D^{\alpha} \xi_{22}^{1}(t)= & -1.6026 \xi_{22}^{1}(t)+0.1795 Z+5.2484 \\
& +0.0225 I+3 f_{2}+d(t), \\
\eta_{21}^{1}(t)= & \xi_{21}^{1}(t) \\
\eta_{22}^{1}(t)= & \xi_{22}^{1}(t)
\end{aligned}
$$

and

$$
\begin{aligned}
D^{\alpha} \widehat{\xi}_{21}^{1}(t)= & -1.6026 \widehat{\xi}_{21}^{1}(t)+0.1795 Z+5.2484 \\
& +0.0225 I+0.1 \widehat{d}(t) \\
& +3.6\left(\eta_{21}^{1}(t)-\widehat{\eta}_{21}^{1}(t)\right), \\
D^{\alpha} \widehat{\xi}_{22}^{1}(t)= & -1.6026 \widehat{\xi}_{22}^{1}(t)+0.1795 Z+5.2484 \\
& +0.0225 I+3 \widehat{f}_{2}+\widehat{d}(t) \\
& +3.5\left(\eta_{22}^{1}(t)-\widehat{\eta}_{22}^{1}(t)\right), \\
\hat{\eta}_{21}^{1}(t)= & \widehat{\xi}_{21}^{1}(t), \\
\hat{\eta}_{22}^{1}(t)= & \widehat{\xi}_{22}^{1}(t),
\end{aligned}
$$

where

$$
\begin{aligned}
& \widehat{f}_{2}= \begin{cases}\rho \frac{h_{1}(t)}{h(t)}, & h(t) \neq 0 ; \\
0, & h(t)=0,\end{cases} \\
& \widehat{d}= \begin{cases}\rho \frac{h_{2}(t)}{h(t)}, & h(t) \neq 0 ; \\
0, & h(t)=0\end{cases}
\end{aligned}
$$

and

$$
\begin{aligned}
& h_{1}(t)=9\left(\xi_{21}^{1}-\widehat{\xi}_{21}^{1}\right)+4\left(\xi_{22}^{1}-\widehat{\xi}_{22}^{1}\right), \\
& h_{2}(t)=4\left(\xi_{21}^{1}-\widehat{\xi}_{21}^{1}\right)+\left(0.3+\frac{4}{3}\right)\left(\xi_{22}^{1}-\widehat{\xi}_{22}^{1}\right), \\
& h(t)=\sqrt{h_{1}(t)^{2}+h_{2}(t)^{2}} .
\end{aligned}
$$



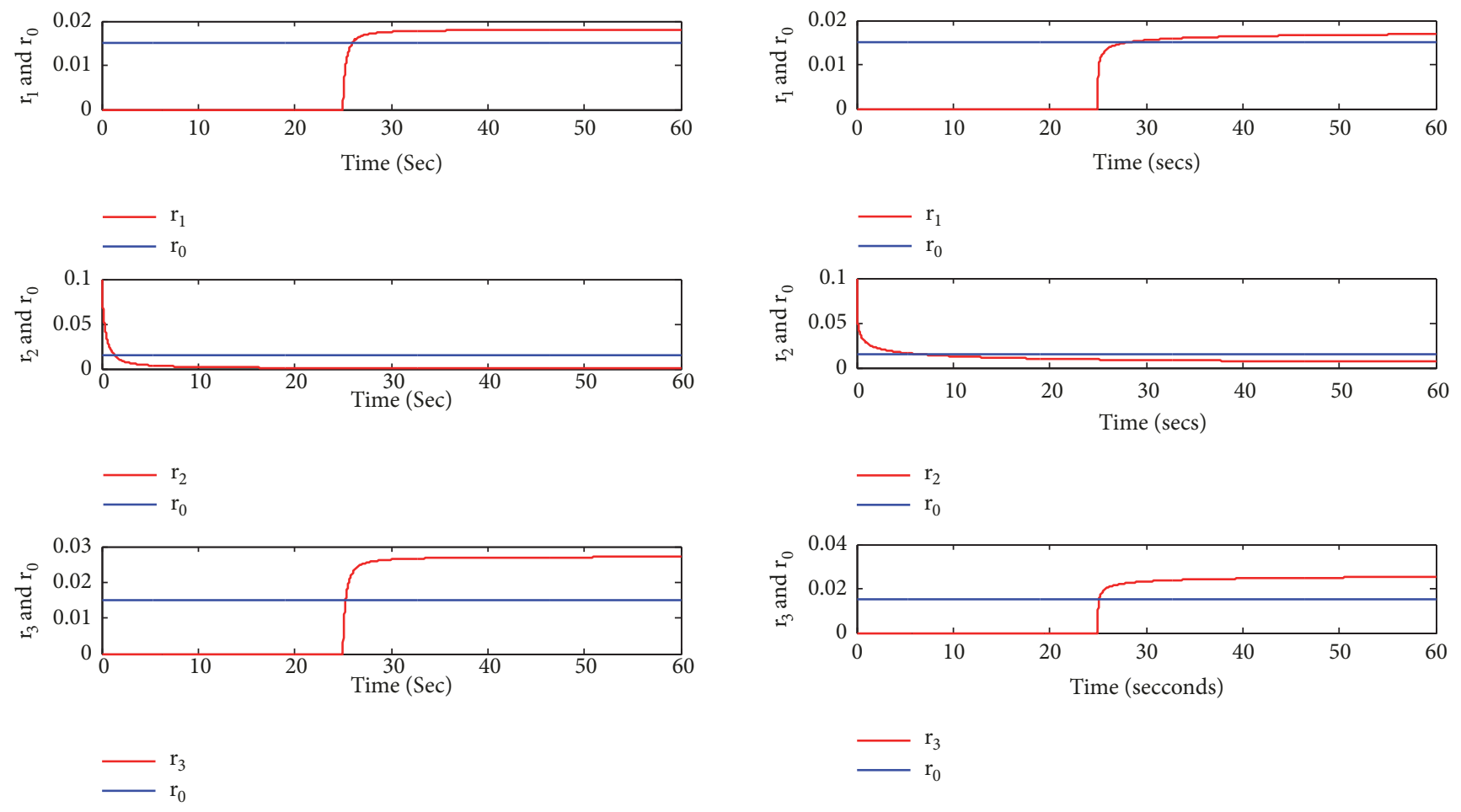

FIgURE 1: Fault detection and isolation when $\rho=0.25, \alpha=0.8$.

To illustrate the performance of the above strategy, the Adams-Bashforth-Moulton predictor-corrector method is used to calculate the fractional system, and the detailed instructions of this algorithm are available in $[47,48]$ based on [49]. Next, the simulations are performed while parameter $\rho$ and the commensurate order $\alpha$ are changed.

Fault $f_{2}(t)$ is caused by a change of internal resistance of the battery and occurs at time instant 25 seconds. Figures 1 and 2 show the evolution of fault-detection residuals $r_{i}(t), i=$ $1,2,3$, and the chosen threshold $r_{0}$ where the horizontal bold lines are thresholds with $\rho=0.25, \alpha=0.8$, and $\rho=100, \alpha=$ 0.4 . It can be seen indeed in the figures that $f_{2}(t)$ is detected at $25 \mathrm{~s}$ because $r_{1}$ and $r_{3}$ go beyond the threshold $r_{0}$ after 25 seconds, and $r_{2}$ is well below the threshold $r_{0}$. Moreover the evolution of fault-detection residuals $r_{1}, r_{2}$, and $r_{3}$ is the same when parameters $\rho$ and $\alpha$ are changed.

Figures 3 and 4 show the evolution of the system states (terminal voltage) and their estimations with the fault estimate that is the second subsystem $(i=2)$ with $\rho=0.25, \alpha=$ 0.8 and $\rho=100, \alpha=0.4$. Although parameters $\rho$ and $\alpha$ are changed, it is noted that each state estimation error is Mittag-Leffler stable because the estimation curve is varying along its own state curve in a small bounded interval and the evolution of the curves has not been affected by the changing parameters. At time instant 25 seconds, $\xi_{22}^{1}(t)$ and its estimation start to identify the isolated fault accurately in Figures 3 and 4 .

To illustrate the advantages of our proposed method, the simulations are performed on the integer-order modeling of the three-cell battery string [1]. Figure 5 shows the evolution of state and its estimate with the fault estimate system by

FIGURE 2: Fault detection and isolation when $\rho=100, \alpha=0.4$.

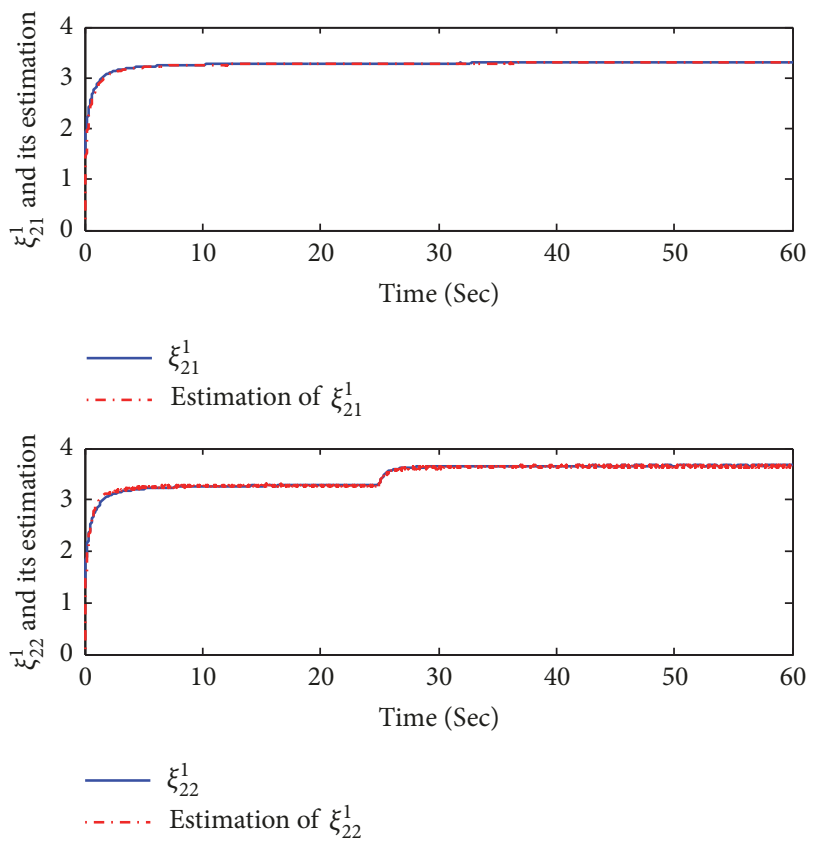

FIGURE 3: System state and estimate with the fault estimate when $\rho=0.25, \alpha=0.8$.

applying the integer-order modeling in [1]. Compared with our proposed method, it can be found that the state and its estimation with the fault estimate start to identify the isolated fault at time instant 25 seconds. But at instant 50 seconds, dynamic curve of estimation with the fault estimate jumps again. The estimation value deviates from state value at the instant too. But the evolution of curves in Figures 3 and 4 

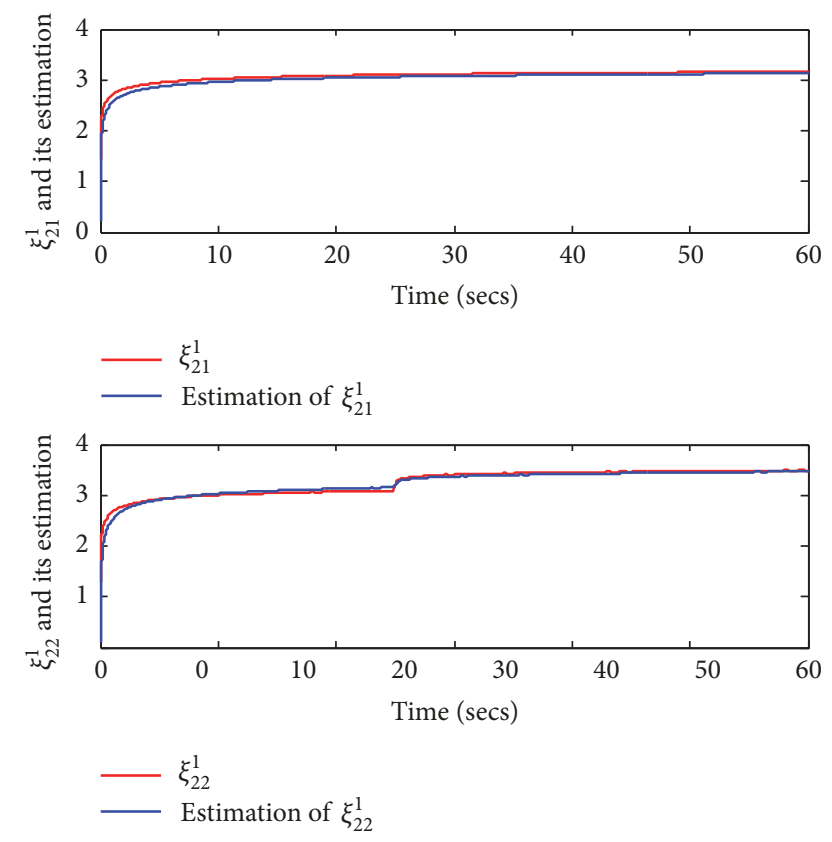

Figure 4: System state and estimate with the fault estimate when $\rho=100, \alpha=0.4$.
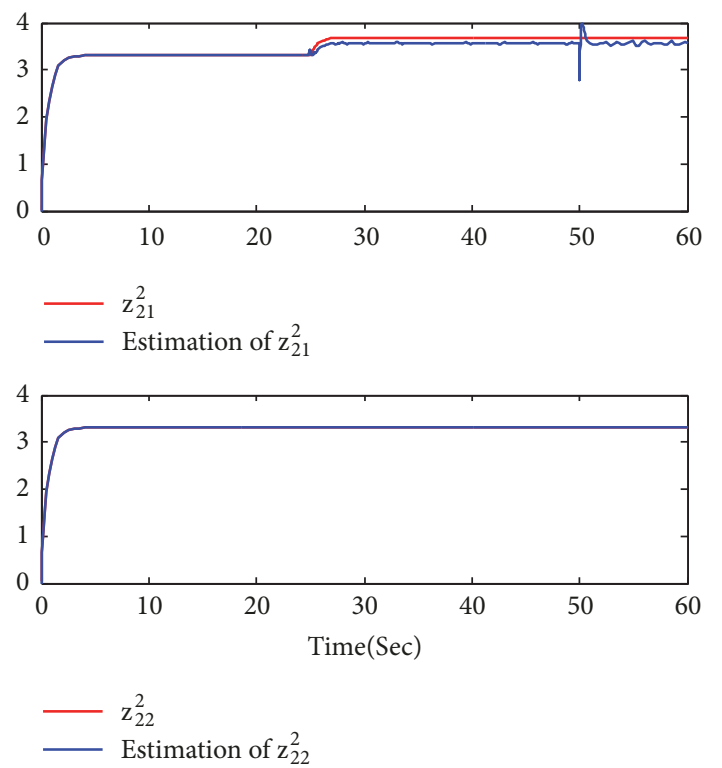

Figure 5: System state and estimate with the fault estimate of the integer-order modeling.

is changed smoothly except at time instant 25 seconds when fault $f_{2}$ occurs. It is shown that our proposed method of fractional-order modeling has even better results than that of integer-order modeling.

\section{Conclusions}

Fault diagnosis and estimation problem have been studied for a fractional-order system with applications to Lithium-ion battery cell. Sources of fault and system uncertainties are first separated into two subsystems through a transformation on the model of the system. A fractional-order Luenberger observer is designed to generate a fault-detection residual and faults are then easily detected and isolated. Secondly, a fractional-order sliding mode observer is constructed to provide an estimate of the isolated fault; hence, simultaneous fault detection, isolation, and estimation is accomplished. The effectiveness of the proposed strategy is demonstrated on a three-cell battery string. The proposed strategy is completely built on a new framework. Properties of fractional-order systems and Mittag-Leffler stability are used to describe the estimation error of observers by the Lyapunov directed method. The stability in the sense of Mittag-Leffler embodies properties of fractional calculus.

\section{Data Availability}

The data supporting the conclusions of our manuscript are some open access articles that have been properly cited, and the readers can easily obtain these articles to verify the conclusions, replicate the analysis, and conduct secondary analyses. Therefore, we do not create a publicly available data repository.

\section{Conflicts of Interest}

The authors declare that they have no conflicts of interest.

\section{Acknowledgments}

This work was supported in part by the Natural Science Foundation of China under Grant 61873144 and Shandong Province under Grant ZR2017MF016 and in the other part by the Natural Science Fundamental Research Project of Jiangsu Colleges and Universities under Grant 17KJB120002, the Natural Science Foundation of Jiangsu Province under Grant BK20170291, and the National Natural Science Foundation of China under Grant 61703059.

\section{References}

[1] W. Chen, W.-T. Chen, M. Saif, M.-F. Li, and H. Wu, "Simultaneous fault isolation and estimation of lithium-ion batteries via synthesized design of Luenberger and learning observers," IEEE Transactions on Control Systems Technology, vol. 22, no. 1, pp. 290-298, 2014.

[2] R. Razavi-Far, S. Chakrabarti, and M. Saif, "Multi-step-ahead prediction techniques for Lithium-ion batteries condition prognosis," in Proceedings of the 2016 IEEE International Conference on Systems, Man, and Cybernetics (SMC), pp. 004675-004680, Budapest, Hungary, October 2016.

[3] M. F. Samadi and M. Saif, "Identifiability analysis of an electrochemical model of li-ion battery," in Proceedings of the 2016 American Control Conference, ACC 2016, pp. 3107-3112, Boston, Mass, USA, July 2016.

[4] M. F. Samadi and M. Saif, "Health monitoring of Li-ion batteries: A particle filtering approach," in Proceedings of the 2015 IEEE 
24th International Symposium on Industrial Electronics (ISIE), pp. 831-836, Buzios, Rio de Janeiro, Brazil, June 2015.

[5] M. F. Samadi and M. Saif, "State-Space Modeling and Observer Design of Li-Ion Batteries Using Takagi-Sugeno Fuzzy System," IEEE Transactions on Control Systems Technology, vol. 25, no. 1, pp. 301-308, 2017.

[6] S. M. M. Alavi, M. F. Samadi, and M. Saif, "Diagnostics in lithium-ion batteries: Challenging issues and recent achievements," in Integration of Practice-Oriented Knowledge Technology: Trends and Prospectives, pp. 277-291, Springer, Berlin, Germany, 2013.

[7] M. F. Samadi and M. Saif, "Integrated battery management system," in Integrated Systems: Innovations and Applications, M. Fathi, Ed., pp. 173-193, Springer, Cham, Switzerland, 2015.

[8] T. Shen, J. Xin, and J. Huang, "Time-space fractional stochastic Ginzburg-Landau equation driven by gaussian white noise," Stochastic Analysis and Applications, vol. 36, no. 1, pp. 103-113, 2018.

[9] I. Podlubny, Fractional Differential Equations, vol. 198 of Mathematics in Science and Engineering, Academic Press, San Diego, Calif, USA, 1999.

[10] R. L. Magin, Fractional Calculus in Bioengineering, Begell House, Redding, Calif, USA, 2006.

[11] N. Laskin, "Fractional market dynamics," Physica A: Statistical Mechanics and its Applications, vol. 287, no. 3-4, pp. 482-492, 2000.

[12] K. M. Zhang, "On a sign-changing solution for some fractional differential equations," Boundary Value Problems, vol. 2017, no. 59, 8 pages, 2017.

[13] J. Liu and Z. Zhao, "Multiple solutions for impulsive problems with non-autonomous perturbations," Applied Mathematics Letters, vol. 64, pp. 143-149, 2017.

[14] Q. Feng and F. Meng, “Traveling wave solutions for fractional partial differential equations arising in mathematical physics by an improved fractional Jacobi elliptic equation method," Mathematical Methods in the Applied Sciences, vol. 40, no. 10, pp. 3676-3686, 2017.

[15] A. M. El-Sayed, "Fractional-order diffusion-wave equation," International Journal of Theoretical Physics, vol. 35, no. 2, pp. 311322, 1996.

[16] Y. Guan, Z. Zhao, and X. Lin, "On the existence of positive solutions and negative solutions of singular fractional differential equations via global bifurcation techniques," Boundary Value Problems, vol. 2016, no. 1, article 141, 2016.

[17] H. Liu and F. Meng, "Some new generalized Volterra-Fredholm type discrete fractional sum inequalities and their applications," Journal of Inequalities and Applications, vol. 2016, no. 1, article 213, 2016.

[18] J. Sabatier, M. Aoun, A. Oustaloup, G. Grégoire, F. Ragot, and P. Roy, "Fractional system identification for lead acid battery state of charge estimation," Signal Processing, vol. 86, no. 10, pp. 26452657, 2006.

[19] J. Sabatier, M. Cugnet, S. Laruelle et al., "A fractional order model for lead-acid battery crankability estimation," Communications in Nonlinear Science and Numerical Simulation, vol. 15, no. 5, pp. 1308-1317, 2010.

[20] H. Wu, S. Yuan, and C. Yin, "A Lithium-Ion battery fractional order state space model and its time domain system identification," in Proceedings of the FISITA 2012 World Automotive Congress, vol. 192 of Lecture Notes in Electrical Engineering, pp. 795-805, Springer Berlin Heidelberg, Berlin, Germany, 2013.
[21] C. Zou, L. Zhang, X. Hu, Z. Wang, T. Wik, and M. Pecht, "A review of fractional-order techniques applied to lithium-ion batteries, lead-acid batteries, and supercapacitors," Journal of Power Sources, vol. 390, pp. 286-296, 2018.

[22] S. M. M. Alavi, C. R. Birkl, and D. A. Howey, “Time-domain fitting of battery electrochemical impedance models," Journal of Power Sources, vol. 288, pp. 345-352, 2015.

[23] J. Zhang and J. Lee, "A review on prognostics and health monitoring of Li-ion battery," Journal of Power Sources, vol. 196, no. 15 , pp. 6007-6014, 2011.

[24] A. T. Stamps, C. E. Holland, R. E. White, and E. P. Gatzke, "Analysis of capacity fade in a lithium ion battery," Journal of Power Sources, vol. 150, no. 1-2, pp. 229-239, 2005.

[25] K. W. E. Cheng, B. P. Divakar, H. Wu, K. Ding, and H. F. Ho, "Battery-management system (BMS) and SOC development for electrical vehicles," IEEE Transactions on Vehicular Technology, vol. 60, no. 1, pp. 76-88, 2011.

[26] A. Sidhu, A. Izadian, and S. Anwar, "Adaptive nonlinear modelbased fault diagnosis of li-ion batteries," IEEE Transactions on Industrial Electronics, vol. 62, no. 2, pp. 1002-1011, 2015.

[27] M. U. Cuma and T. Koroglu, "A comprehensive review on estimation strategies used in hybrid and battery electric vehicles," Renewable \& Sustainable Energy Reviews, vol. 42, pp. 517-531, 2015.

[28] S. Dey and B. Ayalew, "A diagnostic scheme for detection, isolation and estimation of electrochemical faults in LithiumIon cells," in Proceedings of the ASME 2015 Dynamic Systems and Control Conference, 10 pages, Columbus, Ohio, USA.

[29] S. Kong, M. Saif, and B. Liu, "Observer design for a class of nonlinear fractional-order systems with unknown input," Journal of The Franklin Institute, vol. 354, no. 13, pp. 5503-5518, 2017.

[30] E. A. Boroujeni and H. R. Momeni, "Non-fragile nonlinear fractional order observer design for a class of nonlinear fractional order systems," Signal Processing, vol. 92, no. 10, pp. 2365-2370, 2012.

[31] I. N'Doye, M. Darouach, H. Voos, and M. Zasadzinski, "Design of unknown input fractional-order observers for fractionalorder systems," International Journal of Applied Mathematics and Computer Science, vol. 23, no. 3, pp. 491-500, 2013.

[32] Y.-H. Lan and Y. Zhou, "Non-fragile observer-based robust control for a class of fractional-order nonlinear systems," Systems \& Control Letters, vol. 62, no. 12, pp. 1143-1150, 2013.

[33] P. Gong and W. Lan, "Adaptive robust tracking control for uncertain nonlinear fractional-order multi-agent systems with directed topologies," Automatica, vol. 92, pp. 92-99, 2018.

[34] A. Aribi, C. Farges, M. Aoun, P. Melchior, S. Najar, and M. N. Abdelkrim, "Fault detection based on fractional order models: Application to diagnosis of thermal systems," Communications in Nonlinear Science and Numerical Simulation, vol. 19, no. 10, pp. 3679-3693, 2014.

[35] I. N'Doye and T.-M. Laleg-Kirati, "Fractional-order adaptive fault estimation for a class of nonlinear fractional-order systems," in Proceedings of the 2015 American Control Conference, ACC 2015, pp. 3804-3809, Chicago, Ill, USA, July 2015.

[36] J. C. Trigeassou, N. Maamri, J. Sabatier, and A. Oustaloup, "A Lyapunov approach to the stability of fractional differential equations," Signal Processing, vol. 91, no. 3, pp. 437-445, 2011.

[37] X. Lin, Z. Zhao, and Y. Guan, "Iterative Technology in a Singular Fractional Boundary Value Problem with $q$-Difference," Applied Mathematics, vol. 07, no. 01, pp. 91-97, 2016. 
[38] J. C. Trigeassou, N. Maamri, J. Sabatier, and A. Oustaloup, "State variables and transients of fractional order differential systems," Computers \& Mathematics with Applications, vol. 64, no. 10, pp. 3117-3140, 2012.

[39] S. Kong, M. Saif, and H. Zhang, "Optimal filtering for Itostochastic continuous-time systems with multiple delayed measurements," Institute of Electrical and Electronics Engineers Transactions on Automatic Control, vol. 58, no. 7, pp. 1872-1877, 2013.

[40] D. Wang, J. Zhang, and X. Wang, "Synchronization of uncertain fractional-order chaotic systems with disturbance based on a fractional terminal sliding mode controller," Chinese Physics $B$, vol. 22, no. 4, Article ID 040507, pp. 1-7, 2013.

[41] M. Corless and J. Tu, "State and input estimation for a class of uncertain systems," Automatica, vol. 34, no. 6, pp. 757-764, 1998.

[42] P. Kudva, N. Viswanadham, and A. Ramakrishna, "Observers for linear systems with unknown inputs," IEEE Transactions on Automatic Control, vol. 25, no. 1, pp. 113-115, 1980.

[43] D. Matignon and B. Andréa-Novel, "Observer-based for fractional differential systems," in Proceedings of the IEEE Conference on Decision and Control, pp. 4967-4972, San Diego, Calif, USA, 1997.

[44] Y. Li, Y. Chen, and I. Podlubny, "Mittag-Leffler stability of fractional order nonlinear dynamic systems," Automatica, vol. 45, no. 8, pp. 1965-1969, 2009.

[45] J. M. Yu, H. Hu, S. B. Zhou, and X. R. Lin, "Generalized MittagLeffler stability of multi-variables fractional order nonlinear systems," Automatica, vol. 49, no. 6, pp. 1798-1803, 2013.

[46] M. A. Duarte-Mermoud, N. Aguila-Camacho, J. A. Gallegos, and R. Castro-Linares, "Using general quadratic LYApunov functions to prove LYApunov uniform stability for fractional order systems," Communications in Nonlinear Science and Numerical Simulation, vol. 22, no. 1-3, pp. 650-659, 2015.

[47] K. Diethelm, N. J. Ford, and A. D. Freed, "A predictor-corrector approach for the numerical solution of fractional differential equations," Nonlinear Dynamics, vol. 29, no. 1-4, pp. 3-22, 2002.

[48] K. Diethelm, N. J. Ford, and A. D. Freed, "Detailed error analysis for a fractional Adams method," Numerical Algorithms, vol. 36, no. 1, pp. 31-52, 2004.

[49] P. Ivo, Fractional-Order Nonlinear Systems: Modeling, Analysis and Simulation, Springer, Berlin, Germany, 2011. 


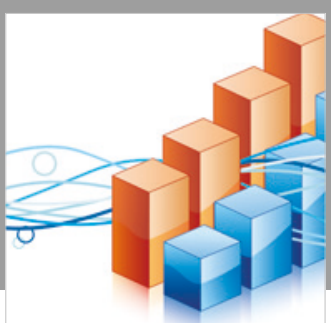

Advances in

Operations Research

\section{-n-m}
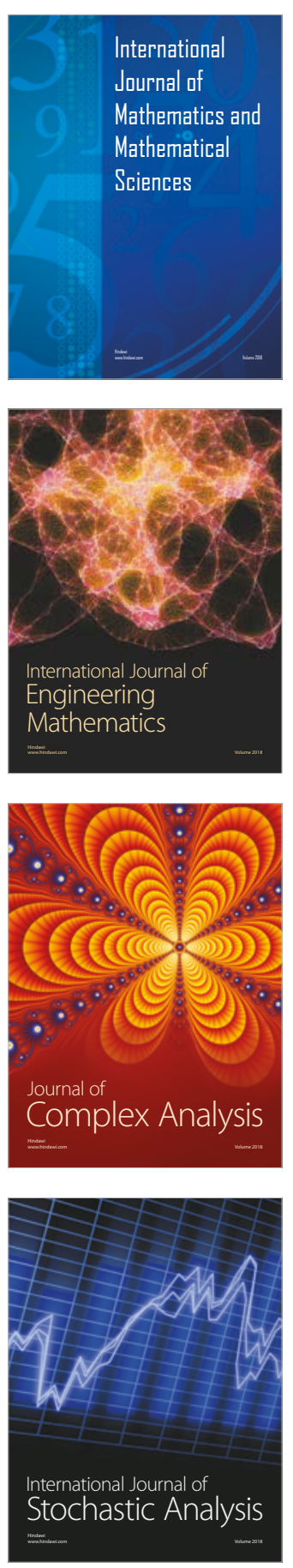
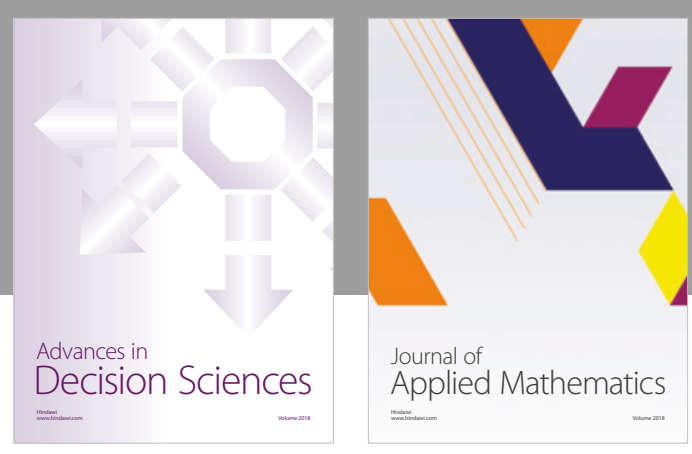

Journal of

Applied Mathematics
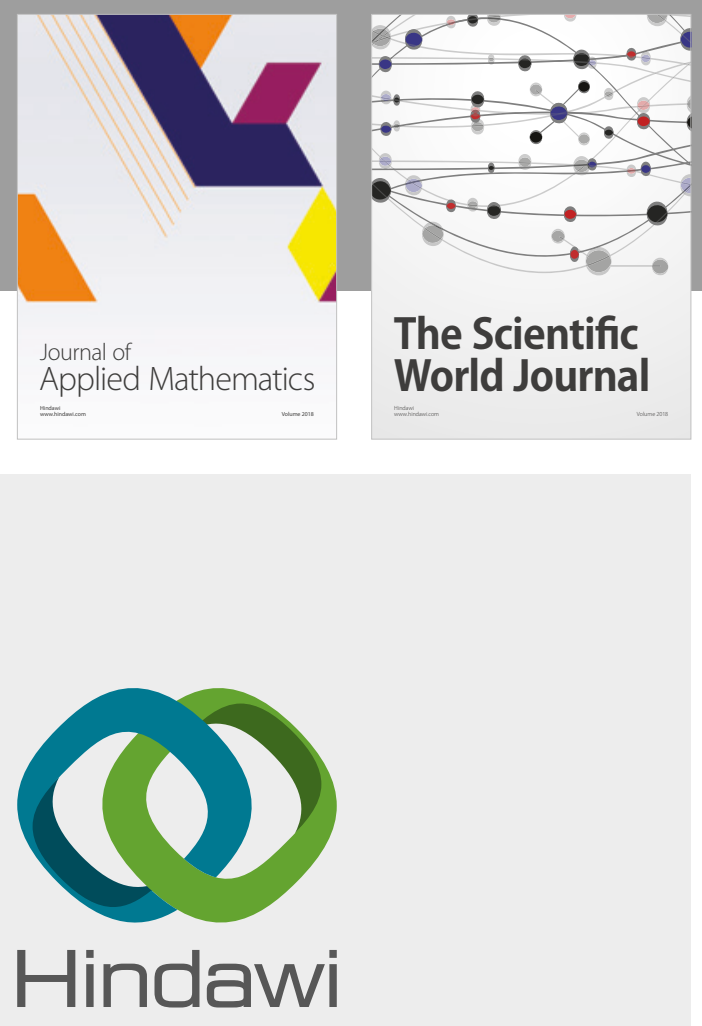

Submit your manuscripts at

www.hindawi.com

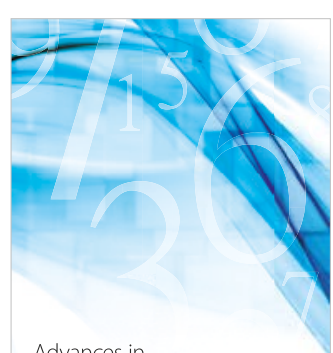

Advances in
Numerical Analysis
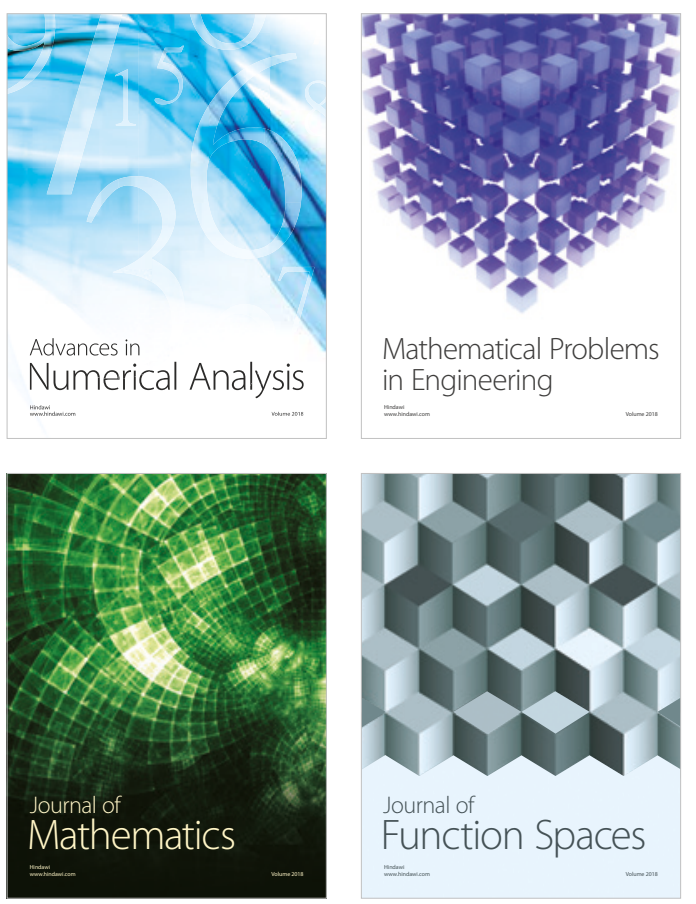

Mathematical Problems in Engineering

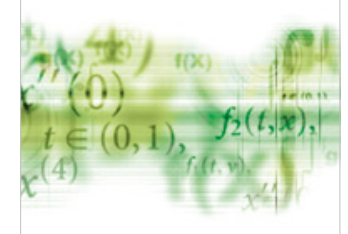

International Journal of

Differential Equations

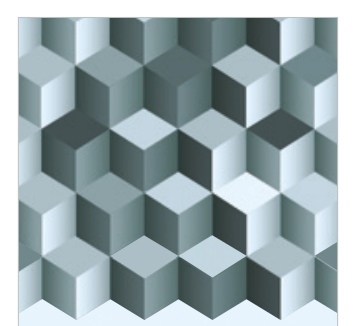

Journal of

Function Spaces

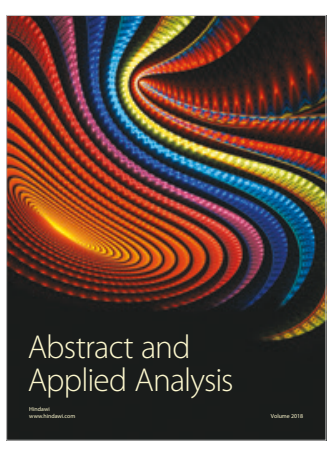

The Scientific

World Journal

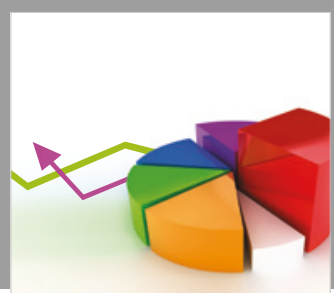

Journal of

Probability and Statistics
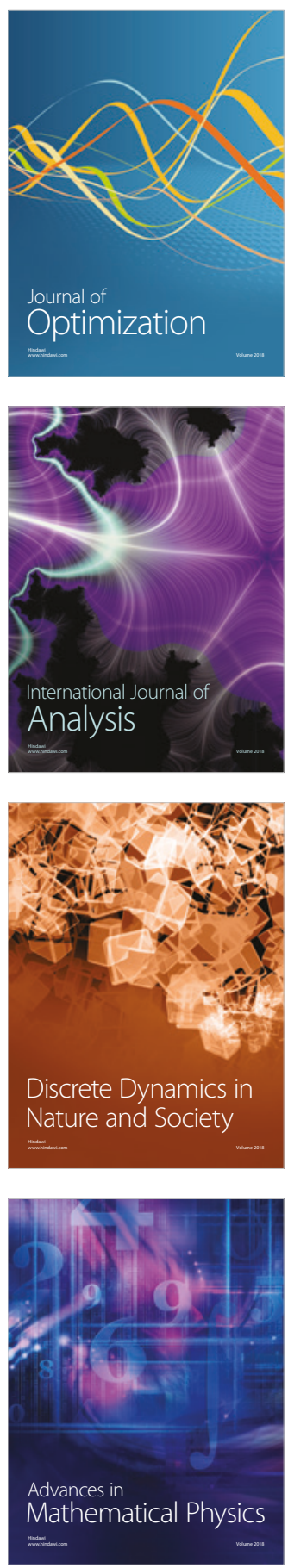